\title{
Budgets of disturbances energy for nozzle flows at subsonic and choked regimes
}

\author{
Maxime Huet \\ ONERA - The French Aerospace Lab \\ F-92322, Châtillon, France \\ Email: maxime.huet@onera.fr
}

\begin{abstract}
The noise generated by the passage of acoustic and entropy perturbations through subsonic and choked nozzle flows is investigated numerically using an energetic approach. Low-order models are used to reproduce the experimental results of the Hot Acoustic Test rig (HAT) of DLR and energy budgets are performed to characterize the reflection, transmission and dissipation of the fluctuations. Because acoustic and entropy perturbations are present in the flow in the general case, classical acoustic energy budgets cannot be used and the disturbances energy budgets proposed by Myers (J. Fluid Mech. 226 (1991) 383-400) are used instead. Numerical results are in very good agreement with the experiments in terms of acoustic transmission and reflection coefficients. The normal shock present in the diffuser for choked regimes is shown to attenuate the scattered acoustic fluctuations, either by pure dissipation effect or by converting a part of the acoustic energy into entropy fluctuations.
\end{abstract}

\section{Introduction}

Increased aircraft operations combined with urban densification around airports lead to the definition of more and more severe environmental regulations [1]. As the dominant noise sources for an aircraft engine, jet and fan noise received an important attention during the last decades. Their relative contribution to global noise has been largely reduced and other sources that were previously masked now emerge. Among these sources, combustion noise which is related to the combustion process becomes now dominant during approach in the medium frequency range, for instance [2]. Combustion noise is classically divided between direct noise and indirect noise [2,3]. Direct noise is the noise generated by the unsteady heat release of the flame. The unsteady turbulent flame also generates entropy and vorticity fluctuations that emit noise when accelerated by the mean flow. The latter is called indirect noise [4-6].

Marble and Candel [7] were among the first to investigate theoretically the generation of indirect combustion noise through a nozzle. In their seminal paper, they proposed analytical solutions in the compact limit (low frequency assumption) and for a linear velocity profile, assuming a quasi-one-dimensional flow (the flow is accelerated by the variation of the section but the flow variables only depend on the axial coordinate). Their work was later extended to deal with the frequency dependance of the generated noise by assuming arbitrary velocity profiles [8-10], nonlinear perturbations (that may occur at low frequencies) [11] and annular ducts more representative of real engines geometries [12], to cite a few recent papers. Experimental evidence of indirect noise generation have been provided by DLR using the Entropy Wave Generator and the Vorticity Wave Generator for subsonic and choked nozzles $[13,14]$, with the entropy noise generation being well recovered numerically and analytically [15-17]. These experiments were complemented recently with the Hot Acoustic Test rig (HAT) facility, designed by DLR in the frame of the European project RECORD to provide additional results such as the noise scattered through the nozzle and the dependence of noise generation on the mean temperature $[18,19]$.

Measurements on the HAT evidenced that the incident acoustic energy was not fully reemitted by the nozzle in choked configurations. A first quantization of this acoustic energy loss was performed by Knobloch et al. [18] considering acoustic energy budgets, but this approach neglects the energy of the entropy perturbations and is thought to overestimate the real dissipation. The objective of the paper is to provide a more accurate evaluation of the scattering of acoustic energy, energy transfer between acoustic and entropy perturbations, and energy dissipation through nozzle flows using low-order models and the disturbances energy of Myers [20]. The disturbance energy balance equation is obtained by recasting the NavierStokes equations and therefore includes all the phenomena present in the flow. It was successfully used by Talei $e t$ al. [21] for hot and cold turbulent jets and was extended to reactive flows by Brear et al. [22] and Giauque et al. [23]. 
The paper is organised as follows. The disturbances energy is first rederived for quasi-one-dimensional flows in the nonlinear regime. A small perturbation assumption is used to exhibit the contribution of the different waves in the flux terms. The HAT facility is then described and the low-order models of ONERA are validated through the reproduction of the experimental results. The acoustic energy loss occurring for choked flows is evidenced. Disturbances energy budgets are performed afterwards to quantize the dissipation occurring in the flow and the contribution of the different perturbations to the global energy budgets. In the last section, the relevance of the disturbances energy budgets for the investigation of a real combustion noise source is illustrated for a model case combustor. Final remarks are then provided in the conclusion.

\section{Disturbances energy for quasi-1D flows}

Quasi-1D flow equations are used to describe the flow variations produced in a duct by a restriction of the section, such as in a nozzle. Acoustic perturbations entering a nozzle are known to be scattered, with a fraction of the incident acoustic energy being reflected and another fraction being transmitted. When entropy perturbations are accelerated by the mean flow, they also generate acoustic waves that propagate in the usptream and downstream directions [7]. Classical acoustic energy budgets only consider energy fluctuations associated with acoustic perturbations but miss the perturbation energy convected by entropy fluctuations. As a consequence, these budgets fail to predict the energy conversion from acoustics to entropy that occurs when the flow is not ideally expended and a shock is present in the diffuser [24], and overpredict the real dissipation occuring in the flow. In this section, a disturbances energy budget considering both acoustic and entropy fluctuations is derived. Assuming small perturbations, the flux terms are linearised to provide the energy associated with each wave entering or leaving the nozzle. This makes possible in particular the evaluation of energy transfer between acoustics and entropy perturbations as well as the energy dissipation caused by the presence of a shock in the diffuser.

\subsection{Nonlinear formulation}

The nonlinear formulation for disturbances energy proposed by Myers [20] is rederived in this section for quasi-onedimensional flows. The flow equations write

$$
\begin{aligned}
\frac{\partial \rho}{\partial t}+\frac{\partial m}{\partial x} & =-m \frac{1}{A} \frac{d A}{d x} \\
\frac{\partial u}{\partial t}+\frac{\partial H}{\partial x}-T \frac{\partial s}{d x} & =0 \\
\frac{\partial \rho s}{\partial t}+\frac{\partial m s}{\partial x} & =-m s \frac{1}{A} \frac{d A}{d x} \\
\frac{\partial}{\partial t}(\rho H-p)+\frac{\partial m H}{\partial x} & =-m H \frac{1}{A} \frac{d A}{d x}
\end{aligned}
$$

with $t$ the time, $x$ the axial coordinate and $A$ the geometry section. $\rho, u, T, s, p$ stand for the density, velocity, temperature, entropy and pressure respectively, $m=\rho u$ is the mass flux and $H=h+\frac{1}{2} u^{2}$ is the specific stagnation enthalpy. Taking (4) $-(\bar{H}-\bar{T} \bar{s}) \times(1)-\bar{T} \times(3)-\bar{m} \times(2)$, where $\overline{(.)}$ stands for a mean variable, it comes

$$
\frac{\partial E}{\partial t}+\frac{\partial W}{\partial x}=-D
$$

where

$$
\begin{aligned}
E & =\rho(H-\bar{H})-\rho \bar{T}(s-\bar{s})-\bar{m}(u-\bar{u})-(p-\bar{p}) \\
W & =(m-\bar{m})[(H-\bar{H})-\bar{T}(s-\bar{s})]+\bar{m}(T-\bar{T})(s-\bar{s}) \\
D & =\underbrace{(s-\bar{s}) m \frac{\partial \bar{T}}{\partial x}-(s-\bar{s}) \bar{m} \frac{\partial T}{\partial x}}_{D_{1}}+\underbrace{W \frac{1}{A} \frac{d A}{d x}}_{D_{2}}
\end{aligned}
$$

with $E$ the disturbances energy per volume unit, $W$ the energy flux per surface unit and $D$ the dissipation per volume unit.

Let us now consider the integration volume $\Omega$ illustrated in Fig. 1 bounded by the inlet, outlet and wall surfaces respectively noted $A_{1}, A_{2}$ and $\partial \Omega_{w}$ (subscripts $(\cdot)_{1}$ and $(\cdot)_{2}$ correspond to values taken at the inlet and outlet of the domain). 


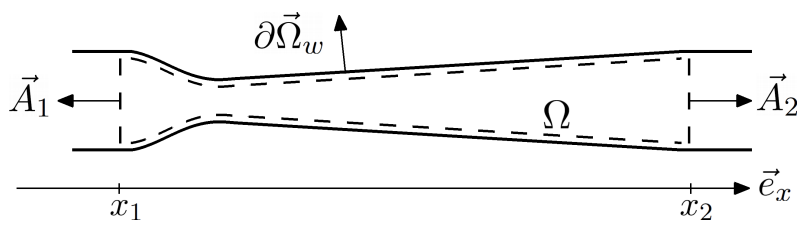

Fig. 1. Integration domain

Integration of Eq. (5) over $\Omega$ leads to, after application of the divergence theorem

$$
\frac{d}{d t}\left(\int_{\Omega} E d \Omega\right) d t+\vec{W}_{1} \overrightarrow{A_{1}}+\vec{W}_{2} \overrightarrow{A_{2}}+\int_{\partial \Omega_{w}} \vec{W} d \vec{S}=-\int_{\Omega} D d \Omega
$$

where $\vec{W}=W \vec{e}_{x}$. It can be shown that $\int_{\partial \Omega_{w}} \vec{W} d \vec{S}=-\int_{\Omega} D_{2} d \Omega$, so that Eq. (9) finally writes

$$
\frac{d \mathcal{E}}{d t}+\mathcal{W}_{1}+\mathcal{W}_{2}=-\mathcal{D}
$$

with

$$
\begin{aligned}
\mathcal{E} & =\int_{\Omega} E d \Omega \\
\mathcal{W}_{1} & =-W_{1} A_{1} \\
\mathcal{W}_{2} & =+W_{2} A_{2} \\
\mathcal{D} & =\int_{\Omega} D_{1} d \Omega
\end{aligned}
$$

In Eq. (10), $\mathcal{D}$ corresponds to the dissipation occuring inside the flow domain. In practice, this dissipation is expected to occur for non-ideally expended supersonic flows, with a normal shock present in the diffuser. Indeed, for such fonfigurations the shock takes energy from the incident perturbations to oscillate.

To end, Eq. (10) is obtained without any assumption for the perturbations. It is in particular valid in the nonlinear regime. However, it cannot be directly derived from Eq. (5) in the presence of a shock because the divergence theorem requires $W$ to be continuously differentiable, which might not be the case through the shock. In that case, Eq. (10) still holds but the dissipation $\mathcal{D}$ does not follow Eq. (14). Moreover, vorticity fluctuations are not considered in this equation due to the quasi-1D hypothesis, even if such fluctuations may be present in real three-dimensional flows [12].

\subsection{Decomposition of the flux term}

In combustion noise, perturbations are generally of small amplitude outside of flames and shocks. This is true in particular at positions $x_{1}$ and $x_{2}$ and Eq. (7) can be linearised to exhibit the contributions of the acoustic and entropy fluctuations to the energy fluxes entering and leaving the integration volume. For low-amplitude perturbations, the energy flux term writes [25]

$$
W=\left(p^{\prime}+\bar{\rho} \bar{u} u^{\prime}\right)\left(u^{\prime}+\frac{\rho^{\prime}}{\bar{\rho}} \bar{u}\right)+\bar{\rho} \bar{u} T^{\prime} s^{\prime}
$$

This equation can be recast to highlight the contributions of the different waves present in the flow

$$
W=W^{+}+W^{-}+W^{s}+W^{+, s}+W^{-, s}
$$

with

$$
W^{ \pm}= \pm \frac{1}{\bar{\rho} \bar{c}}(1 \pm \bar{M})^{2} p^{ \pm 2}
$$




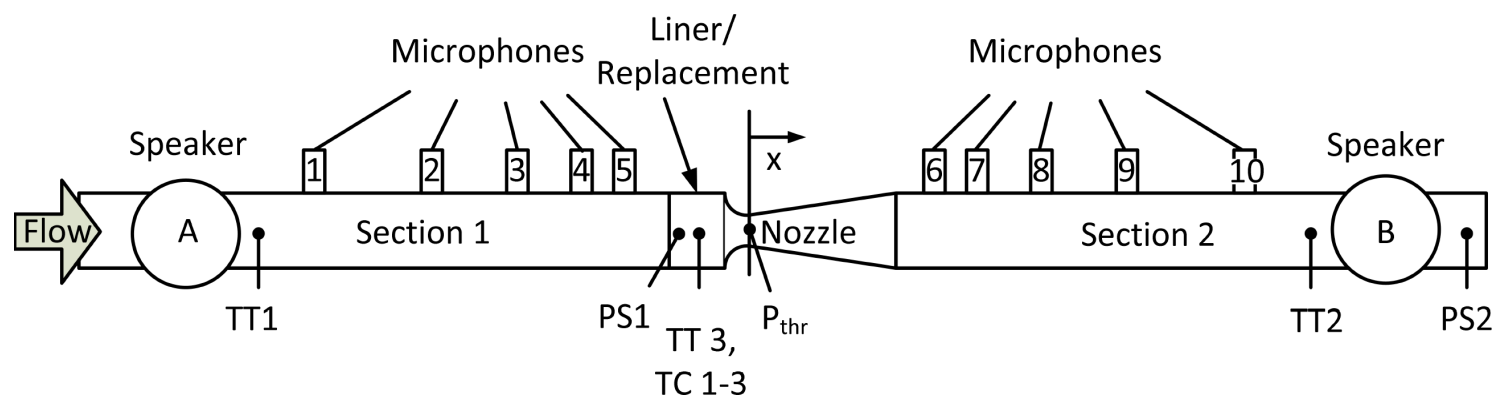

Fig. 2. Sketch of the Hot Acoustic Test rig. $\mathrm{P}_{\text {thr }}$ : pressure at nozzle throat, PS1/2: static pressure, TT: total temperature, TC: fast thermocouple probes [26].

$$
\begin{aligned}
W^{s} & =\frac{\bar{\rho} \bar{u} \bar{T}}{c_{p}} s^{\prime 2} \\
W^{ \pm, s} & =\mp \frac{\bar{u} \bar{M}}{c_{p}} p^{\prime \pm} s^{\prime}
\end{aligned}
$$

where $W^{+}$and $W^{-}$correspond respectively to the energy fluxes per surface unit associated with the progressive and retrograde acoustic waves, $W^{s}$ to the energy flux per surface unit of the entropy wave, $W^{ \pm, s}$ to interaction terms between acoustic and entropy and $p^{\prime+}$ and $p^{\prime-}$ stand for the pressure fluctuations of the acoustic waves $\left(p^{\prime}=p^{\prime+}+p^{\prime-}\right)$. With the notations used, $W_{1}^{+}, W_{2}^{-}$and $W_{1}^{s}$ correspond to the energy fluxes per surface unit associated with the forcing perturbations entering the nozzle, whereas $W_{1}^{-}, W_{2}^{+}$and $W_{2}^{s}$ correspond to the energy fluxes of the waves leaving the nozzle.

\section{Numerical reproduction of HAT}

\subsection{Description of the facility}

The Hot Acoustic Test rig (HAT) is a facility developed by DLR in the frame of the European project RECORD to evaluate the entropy-generated and acoustic-scattered noise by a hot nozzle flow [26,27]. The instrumented part is composed of two ducts with an identical inner diameter of $70 \mathrm{~mm}$ joined by a nozzle with a length of about $400 \mathrm{~mm}$ and whose geometry corresponds to the cross-sectional change of a typical NGV geometry at the high pressure turbine inlet of aero-engines. The mean flow temperature can be varied from ambient to $550^{\circ} \mathrm{C}$ for subsonic and shocked flows. Upstream and downstream ducts are equipped with pressure and temperature probes as well as with an air injection system and loudspeakers to generate entropy and acoustic perturbations. This permits in particular to measure the acoustic transfer functions of the nozzle. A sketch of the facility is visible in Fig. 2. The reader may refer to Knobloch et al. $[18,19]$ for more details on the experimental setup, its instrumentation and the data processing.

\subsection{Low-order modelling}

The modelling of the noise emitted by an accelerated nozzle flow is done with the low-order models MarCan and Sunday of ONERA. MarCan $[9,28]$ is a semi-analytical model that computes the transfer functions of the nozzle for acoustic and entropy forcings in the frequency domain, following the developments of Marble and Candel [7] and Moase et al. [8]. The flow is assumed to be quasi-1D and the velocity profile is piecewise linear. In the harmonic regime, the linearised Euler equations reduce to an hypergeometric differential equation for the nondimensional pressure that is solved numerically. The presence of a shock is modelled using the dynamic jump relations derived from Rankine and Hugoniot. Sunday [9, 11,24] is a flow solver that computes the quasi-1D Euler equations in the time domain using a finite difference approach. Numerical resolution is performed using the standard fourth order Runge-Kutta time scheme and the fourth order optimized finite difference space scheme of Bogey \& Bailly [29]. The adaptative spatial filtering of Bogey et al. [30] is implemented to accurately capture the shocks and non-reflective characteristic boundary conditions are used at the domain boundaries $[31,32]$.

Being a linear harmonic model, MarCan provides a very fast estimation of nozzle transfer functions and was for instance shown to be an efficient tool for shape optimization in order to optimize combustion noise [33]. It is however limited to small amplitude perturbations. On the other side, Sunday makes possible the computation of nonlinear perturbations [11] while exhibiting slightly longer simulation times. It moreover allows to consider non-calorically perfect gaz and to take thermal diffusion into account in the generation of combustion noise [34]. 


\begin{tabular}{lrc}
\hline & Inlet Temperature & Throat Mach number \\
\hline \hline T020_M07 & $20^{\circ} \mathrm{C}$ & 0.7 \\
T100_M07 & $100^{\circ} \mathrm{C}$ & 0.7 \\
T200_M07 & $200^{\circ} \mathrm{C}$ & 0.7 \\
T400_M07 & $400^{\circ} \mathrm{C}$ & 0.7 \\
T020_M10 & $20^{\circ} \mathrm{C}$ & 1.0 \\
T100_M10 & $100^{\circ} \mathrm{C}$ & 1.0 \\
T200_M10 & $200^{\circ} \mathrm{C}$ & 1.0 \\
T400_M10 & $400^{\circ} \mathrm{C}$ & 1.0 \\
\hline
\end{tabular}

Table 1. Experimental data points

\subsection{Reproduction of experimental results}

The eight experimental data points considered in the present study are given in Tab. 1. They correspond to subsonic and choked flows, with the inlet temperature varying between $20^{\circ} \mathrm{C}$ and $400^{\circ} \mathrm{C}$. For choked configuration, the flow is not adapted and a shock forms in the diffuser. In this section, only acoustic forcings are considered.

The acoustic transmission and reflection coefficients of the nozzle are reproduced in Figs. 3 and 4 for the subsonic and choked mean flows, respectively. They correspond to the ratio of the energy fluxes between the generated acoustic wave and the forcing in the linear regime, as defined in Eq. (17). They write

$$
R^{+}=\left|\frac{W_{1}^{-}}{W_{1}^{+}}\right|, R^{-}=\left|\frac{W_{2}^{+}}{W_{2}^{-}}\right|, T^{+}=\left|\frac{A_{2} W_{2}^{+}}{A_{1} W_{1}^{+}}\right|, T^{-}=\left|\frac{A_{1} W_{1}^{-}}{A_{2} W_{2}^{-}}\right|
$$

In these figures, simulated results reproduce the experiments with a good degree of accuracy. In particular, the influence of the frequency and of the mean temperature is very well captured numerically. This illustrates the capacity of the quasi-1D models to reproduce acoustically-forced experimental results as long as planar waves are considered. This assumption limits the validity of the models to low frequencies, for which transverse modes are cut-off. This is however not restrictive for combustion noise, as this noise is associated to low frequencies. It is moreover worth mentioning that this planar wave assumption is not verified for entropy waves because of the radial deformation of the entropy fronts by the mean flow. Modelling entropy-generated noise therefore requires the use of a $2 \mathrm{D}$ model for accurate prediction above a few hundred Hertz [35-37].

More in details, in Fig. 3 (a) the reflection coefficient $R^{+}$is nil in the low frequency limit, quickly rises for frequencies up to $400 \mathrm{~Hz}$ and then slowly decreases. Inlet and outlet sections being identical in the HAT, in the low frequency limit (compact assumption) the nozzle is seen as a straight duct by the acoustic waves and no reflections occur [7]. As the frequency increases, the acoustic wavelengths become comparable to the nozzle length and the noise is scattered. Increasing the mean temperature does not change the shape of the transfer function and simply shifts the levels to higher frequencies. As noted by Knobloch et al. [26], all the transfer functions collapse to a single curve using the nondimensional frequency $f^{*}=f L / c^{*}$, see Fig. 5 (a). In this equation, $L$ stands for the nozzle length and $c^{*}$ to the theoretical critical sound velocity at the sonic throat (even though not reached in subsonic configurations), $c^{*}=\sqrt{2 \gamma r T_{i} /(\gamma+1)}$ with $T_{i}$ the stagnation temperature and $\gamma$ the adiabatic coefficient. This frequency shift caused by mean temperature variation can be demonstrated (not reproduced here for the sake of conciseness) by showing that the hypergeometric differential equation used in the low-order model MarCan remains unchanged when the inlet temperature is varied and the Mach number is kept unchanged. This result applies not only to $R^{+}$but to all reflection and transmission coefficients. The transmission coefficient $T^{+}$exhibits variations opposite to $R^{+}$. The acoustic energy is fully transmitted for the low frequencies in accordance with the compact nozzle interpretation, and then rapidly decreases up to $400 \mathrm{~Hz}$ before increasing slowly. Results are similar for $R^{-}$and $T^{-}$. To end, it has been verified that no dissipation occurs for this configuration, $R^{ \pm}+T^{ \pm}=1$, in agreement with the theoretical expectations because there are no entropy fluctuations.

The evolution of the coefficients is different for the choked configurations, see Fig. 4. Considering the upstream acoustic forcing, the compact reflection coefficient $R^{+}$is not nil in the low frequency limit because the nozzle cannot be seen as a straight duct due to the shock. In the compact limit, $R^{+}=0.60$ is in agreement with analytical solutions [8, 24]. This coefficient falls slowly as the frequency increases, which indicates that the nozzle reflects less and less acoustics and becomes almost non reflective at $3000 \mathrm{~Hz}$. On its side, the transmission coefficient is low in the compact limit, $T^{+}=0.09$, 


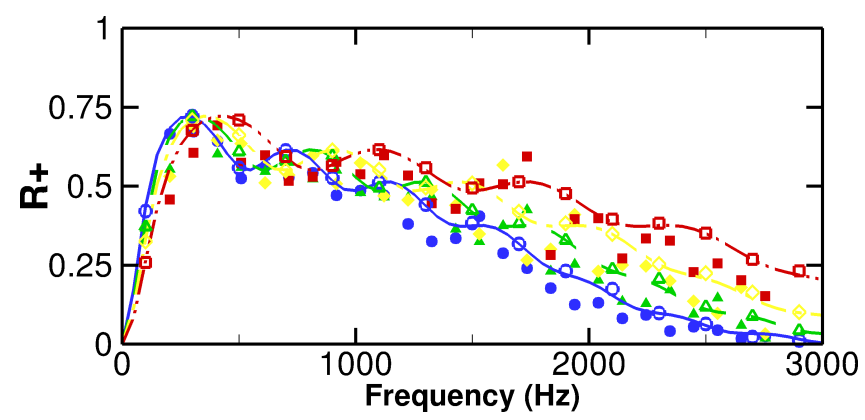

(a) Reflection $R^{+}$

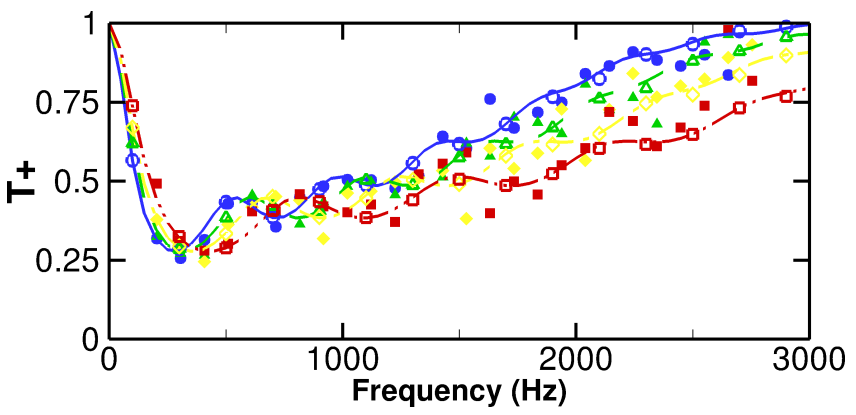

(b) Transmission $T^{+}$

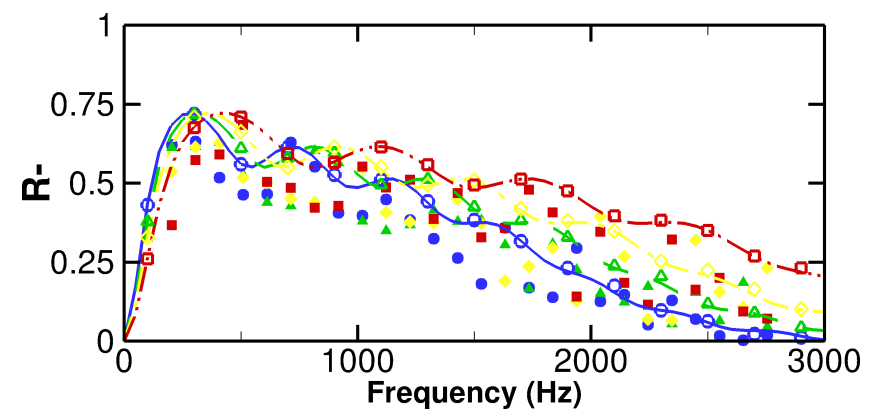

(c) Reflection $R^{-}$

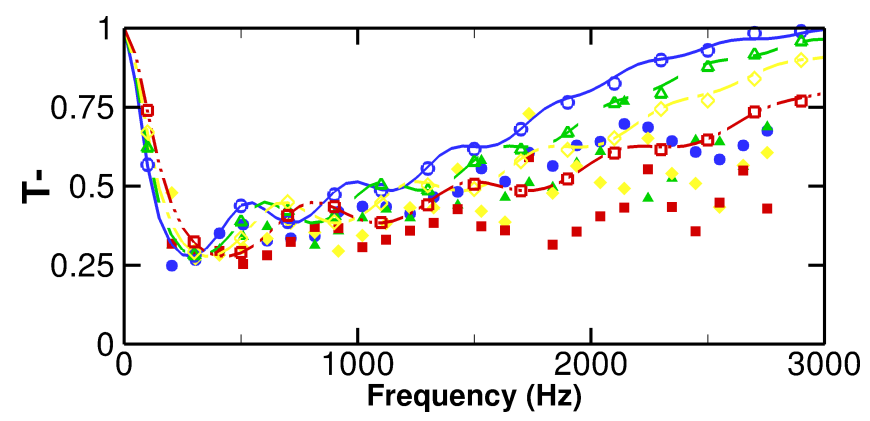

(d) Transmission $T^{-}$

Fig. 3. Experimental and simulated scattering coefficients as a function of the temperature (subsonic case). Experiments: $\bullet 20^{\circ} \mathrm{C}, \Delta 100^{\circ} \mathrm{C}$, $200^{\circ} \mathrm{C}, \square 400^{\circ} \mathrm{C}$; Marcan: $-20^{\circ} \mathrm{C},--100^{\circ} \mathrm{C},-200^{\circ} \mathrm{C},-\cdots 400^{\circ} \mathrm{C}$; Sunday: $\circ 20^{\circ} \mathrm{C}, \triangle 100^{\circ} \mathrm{C}, 200^{\circ} \mathrm{C}, \square 400^{\circ} \mathrm{C}$.

and increases with the frequency so that the nozzle transmits almost all the acoustic energy at $3000 \mathrm{~Hz}$. For a downstream acoustic forcing, the reflection coefficient is large at low frequencies, $R^{-}=0.85$, but rapidly decreases and becomes almost nil above $500 \mathrm{~Hz}$. As the acoustic perturbations coming from the downstream cannot propagate to the upstream duct because of the sonic throat, the transmission coefficient $T^{-}$is nil for all frequencies. The nozzle is therefore silent for frequencies above $500 \mathrm{~Hz}$. Here again, the mean temperature variation only leads to a shift of the frequencies and all the transfer functions collapse using the nondimensional frequency $f^{*}$, see Fig. 5 (b), as the mean Mach number profile is unchanged and the nondimensionalization does not modify the shock relations used in MarCan. From the energetic point of view, for the upstream forcing a small part of the acoustic energy entering the nozzle is not reemitted at low frequencies $\left(R^{+}+T^{+}<1\right)$. 


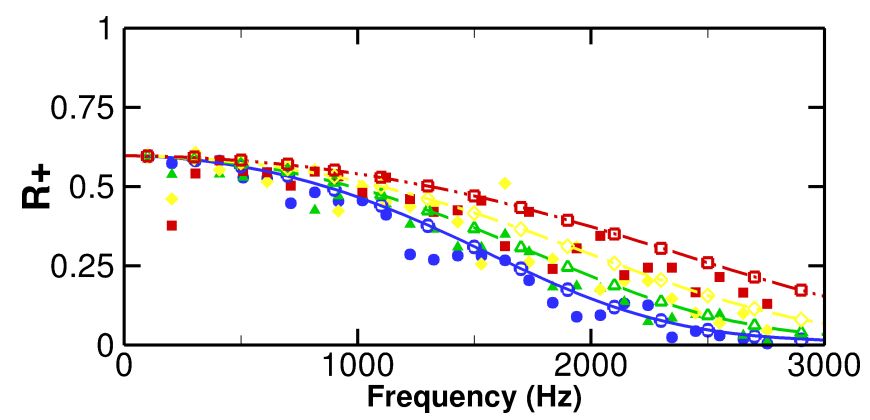

(a) Reflection $R^{+}$

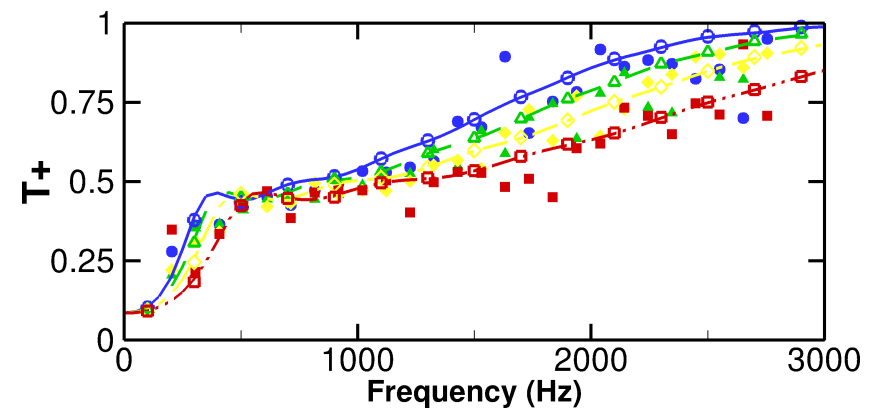

(b) Transmission $T^{+}$

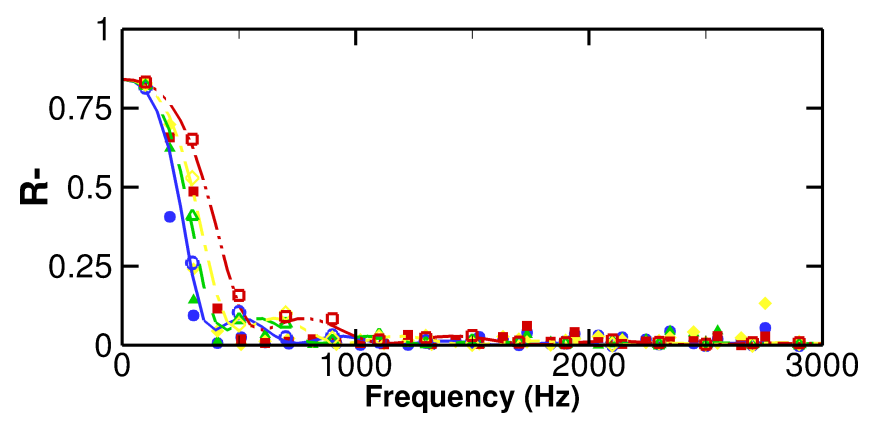

(c) Reflection $R^{-}$

Fig. 4. Experimental and simulated scattering coefficients as a function of the temperature (choked case). See Fig. 3 for the legend.

This observation is more visible for the downstream forcing, where almost no acoustic energy is emitted by the nozzle for frequencies above $500 \mathrm{~Hz}$. This energy is either dissipated by the shock or transferred to entropy fluctuations, as acousticshock interactions are known to generate entropy $[8,24]$. The next section focuses on the disturbances energy budgets and on the contribution of the different waves to the energy balance for the cases T400_M07 and T400_M10 of Tab. 1. This section includes the evaluation of the dissipation and the energy transfer between acoustics and entropy.

\section{Application of disturbances energy to HAT \\ 4.1 Validation of the energy budgets}

In a first step, the closure of the energy budget is verified in the time domain for the case T400_M07. Simulations are run with Sunday. The time evolution of the different terms of the energy budget, Eq. (10), is reproduced in Fig. 6 for a harmonic entropy forcing. In this figure, $\overline{\mathcal{W}}_{1}^{s}$ corresponds to the time-averaged value of the flux of the entropy perturbation over 1 period. The energy budget closes very well, the variation of the energy disturbance in the volume exactly compensates the dissipation and the energy fluxes at the boundaries. The energy budget also closes theoretically for choked configurations, however in the presence of a shock the numerical evaluation of $\mathcal{D}$ diverges because of the spatial derivative of the discontinuous flow fields.

Considering a multi-harmonic forcing (sum of harmonic forcings with random phases), the time-averaged flux and dissipation terms are evaluated in a second step for each frequency for the numerical simulations (Sunday) and the analytical formulation (MarCan). By definition, $d \overline{\mathcal{E}} / d t=0$ so that analytically the dissipation is deduced from the fluxes as $\overline{\mathcal{W}}_{1}+$ $\overline{\mathcal{W}}_{2}+\overline{\mathcal{D}}=0$ 


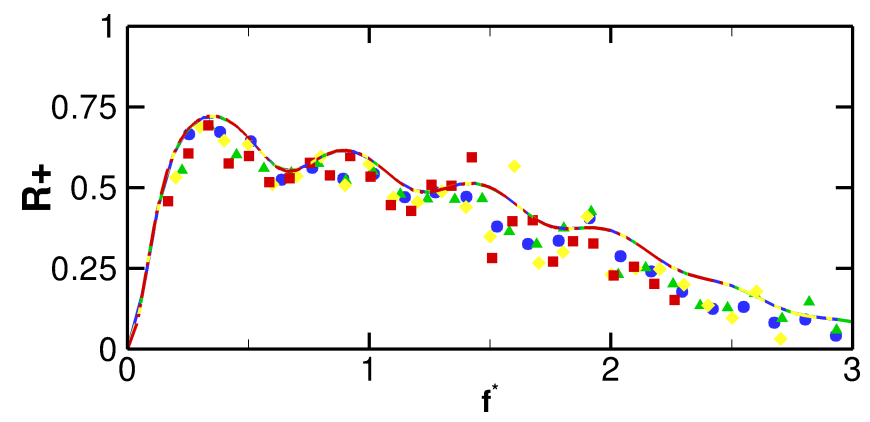

(a) subsonic case

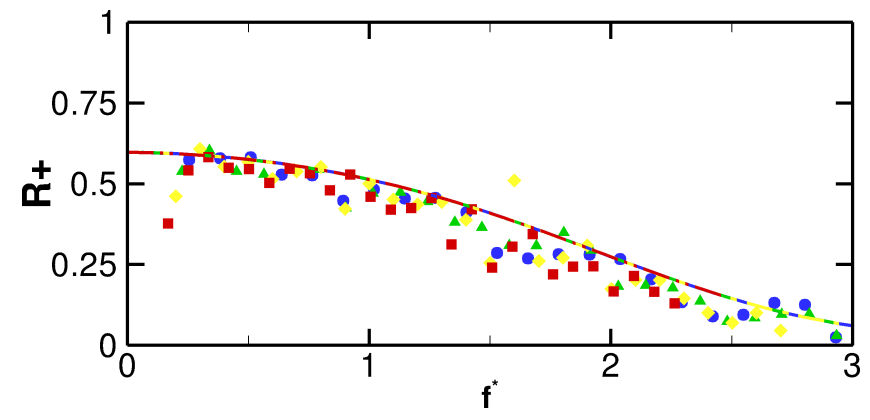

(b) choked case

Fig. 5. Reflection coefficients as a function of the temperature with nondimensionalized frequency. See Fig. 3 for the legend.

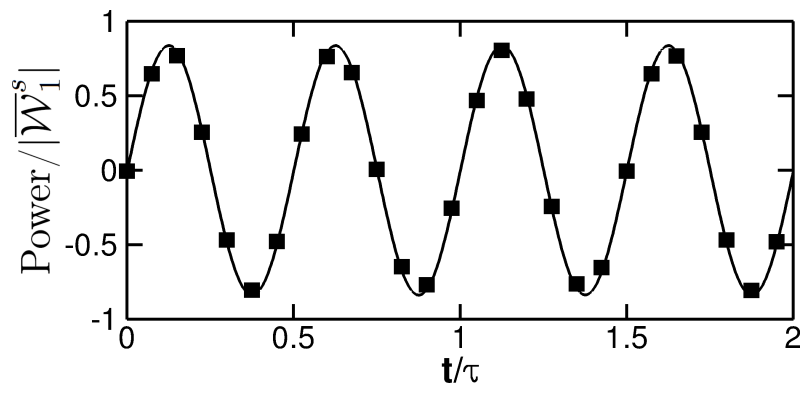

Fig. 6. Time evolution of the different terms of Eq. (10) for nozzle case T400_M07 with harmonic entropy forcing at $3000 \mathrm{~Hz}$ (period $\tau$ ). $d \mathcal{E} / d t, \mathbf{\square}-\left(\mathcal{W}_{1}+\mathcal{W}_{2}+\mathcal{D}\right)$

Figure 7 reproduces the mean disturbances energy budgets for the subsonic configuration nondimensionalized by the mean energy flux of the forcing $\left|\overline{\mathcal{W}}_{f}\right|$, with $\overline{\mathcal{W}}_{f}$ being either $\overline{\mathcal{W}}_{1}^{+}, \overline{\mathcal{W}}_{2}^{-}$or $\overline{\mathcal{W}}_{1}^{s}$ for upstream acoustic forcing, downstream acoustic forcing or entropy forcing, respectively. A negative flux indicates that energy enters the domain through the considered face whereas energy leaves the domain for a positive flux, on average over one period. For an upstream acoustic forcing, Fig. 7 (a), energy globally enters the nozzle by the upstream face and leaves the nozzle by the downstream face. The dissipation is nil whatever the frequency, as expected theoretically. The low values of $\overline{\mathcal{W}}_{1}$ and $\overline{\mathcal{W}}_{2}$ at $400 \mathrm{~Hz}$ are coherent with the large reflection coefficient $R^{+}$and low transmission coefficient $T^{+}$observed in Fig. 3. When the reflection coefficient is large, most of the energy entering the nozzle from the upstream also leaves the nozzle through the upstream face thus reducing the global energy flux through this face and, as a consequence, only a minor part of the energy goes through the nozzle and exits through the downstream face.

Similar observations are made for the downstream acoustic forcing, Fig. 7 (b). The analysis is more complex for the entropy forcing, Fig. 7 (c), as the fluxes involve entropy and acoustic fluctuations. For this case, the upstream flux $\overline{\mathcal{W}}_{1}$ is very close to -1 which indicates that most of the flux corresponds to the entropy wave entering the nozzle. This negative flux is counterbalanced by the positive downstream flux $\overline{\mathcal{W}}_{2}$ corresponding to the acoustic and entropy waves leaving the nozzle. The flow being subsonic, no dissipation is expected to occur physically. The dissipation term is however not nil because of the combined entropy fluctuations and temperature gradients inside the nozzle, see Eq. (8). The negative dissipation term moreover indicates that it produces energy. This result seems to indicate that the expression obtained for the disturbances energy is not totally satisfactory as it does not describe the real dissipation occurring in the flow. The amplitude of the 


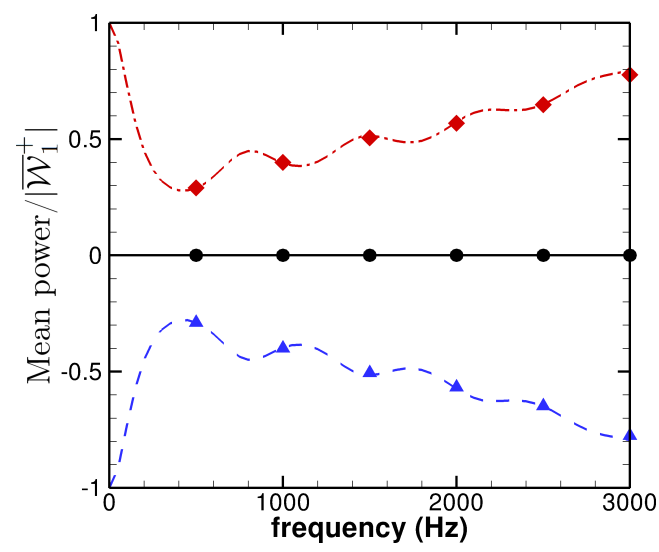

(a) upstream acoustic forcing $P_{1}^{+}$

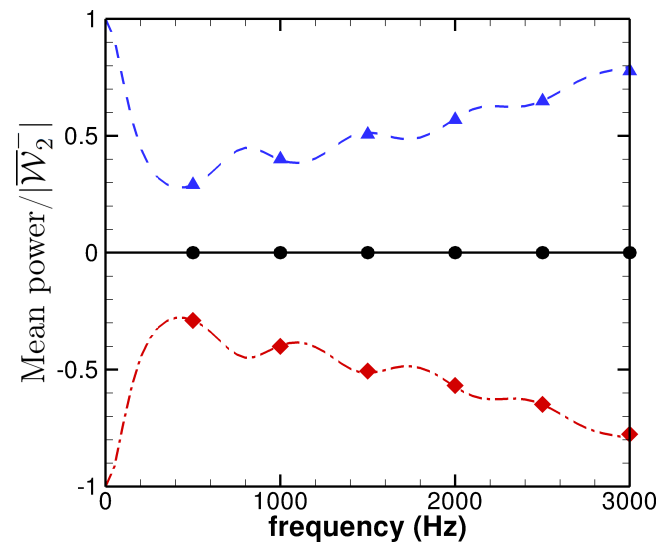

(b) downstream acoustic forcing $P_{2}^{-}$

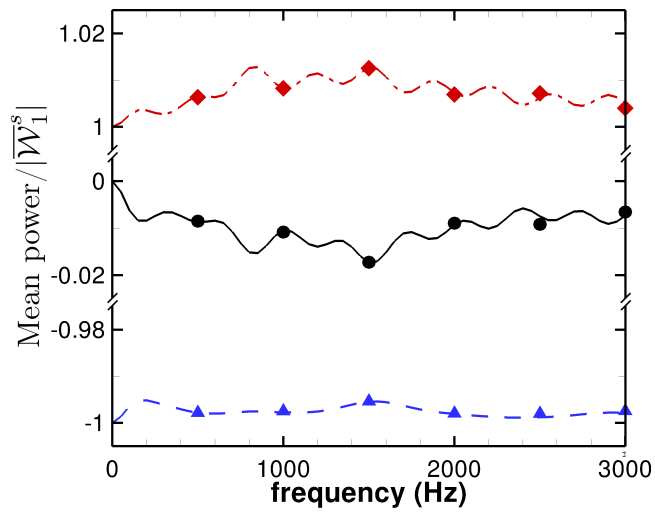

(c) entropy forcing $\sigma_{1}$

Fig. 7. Comparison between numerical and analytical mean disturbances energy budgets for nozzle case T400_M07. Analytical: - $\overline{\mathcal{W}}_{1} /\left|\overline{\mathcal{W}}_{f}\right|,-\cdot \overline{\mathcal{W}}_{2} /\left|\overline{\mathcal{W}}_{f}\right|,-\overline{\mathcal{D}} /\left|\overline{\mathcal{W}}_{f}\right| ;$ Simulation: $\Delta \overline{\mathcal{W}}_{1} /\left|\overline{\mathcal{W}}_{f}\right|, \overline{\mathcal{W}}_{2} /\left|\overline{\mathcal{W}}_{f}\right|, \bullet \overline{\mathcal{D}} /\left|\overline{\mathcal{W}}_{f}\right|$

dissipation is however very low compared to the fluxes and the disturbances energy provides very good trends.

The same analysis is conducted for the choked flow in Fig. 8. With the upstream acoustic forcing, the upstream flux is small at low frequencies because a large part of the acoustics is reflected by the nozzle. The flux tends towards -1 as the frequency increases so that less acoustics is reflected, in agreement with the observations of Fig. 4 (a). The downstream flux increases with the frequency and combines the contributions of acoustic and entropy perturbations. To end, the dissipation is important only for low frequencies and vanishes above $400 \mathrm{~Hz}$. For a downstream acoustic forcing, Fig. 8 (b), all the energy is reflected at low frequencies because the average flux is nil. As the frequency increases, the dissipation grows which explains why the nozzle does not reflect acoustics anymore in Fig. 4 (c). To end with this figure, with an entropy forcing the essential part of the upstream energy corresponds to the incoming entropy fluctuation. Most of the energy is transmitted by the nozzle, except for the low frequencies where a small amount of dissipation is observed. Above $400 \mathrm{~Hz}$, this dissipation 


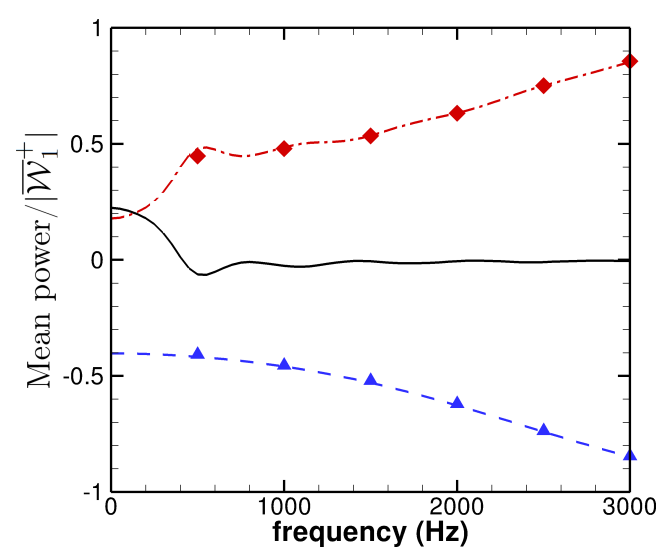

(a) upstream acoustic forcing $P_{1}^{+}$

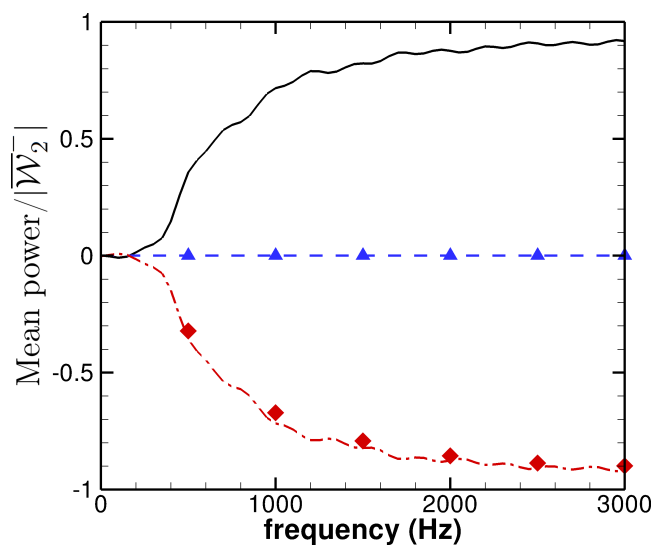

(b) downstream acoustic forcing $P_{2}^{-}$

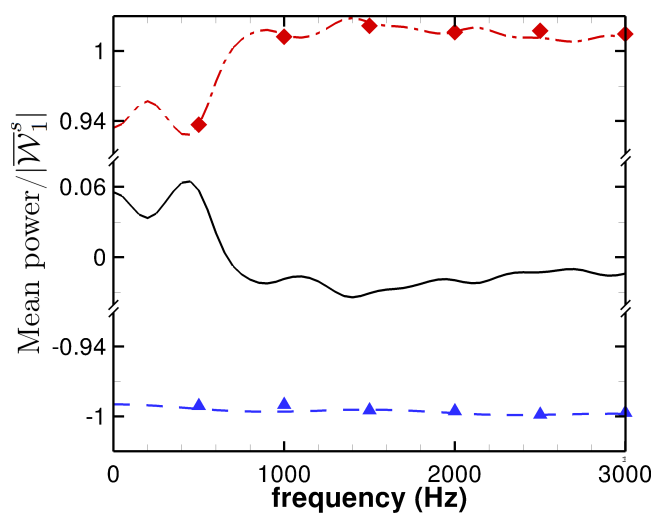

(c) entropy forcing $\sigma_{1}$

Fig. 8. Comparison between numerical and analytical mean disturbances energy budgets for nozzle case T400_M10. Analytical: - $\overline{\mathcal{W}}_{1} /\left|\overline{\mathcal{W}}_{f}\right|,-\cdot \overline{\mathcal{W}}_{2} /\left|\overline{\mathcal{W}}_{f}\right|,-\overline{\mathcal{D}} /\left|\overline{\mathcal{W}}_{f}\right| ;$ Simulation: $\Delta \overline{\mathcal{W}}_{1} /\left|\overline{\mathcal{W}}_{f}\right|, \diamond \overline{\mathcal{W}}_{2} /\left|\overline{\mathcal{W}}_{f}\right|$

becomes negligible. To complete this analysis, it remains to separate the contributions of acoustic and entropy fluctuations to the global upstream and downstream fluxes. This point is discussed in the next section.

\subsection{Contribution of the different waves to the energy balance}

The individual contributions of the different waves to the global fluxes are investigated using Eqs. (17)-(19). The analysis is first performed for the subsonic case and contributions are reproduced in Fig. 9 for the three forcings. For acoustic forcings, entropy fluctuations are nil and only the energy fluxes of the acoustic waves contribute to the energy budget. It is clear from Fig. 9 (a) and (b) that, for acoustic forcings, all the energy is transmitted through the nozzle in the low frequency limit. The maximum energy reflection and minimum energy transmission near $400 \mathrm{~Hz}$ are recovered. These results are in agreement with the previous observations. For the entropy forcing, Fig. 9 (c), it is first observed that the energy

Huet 


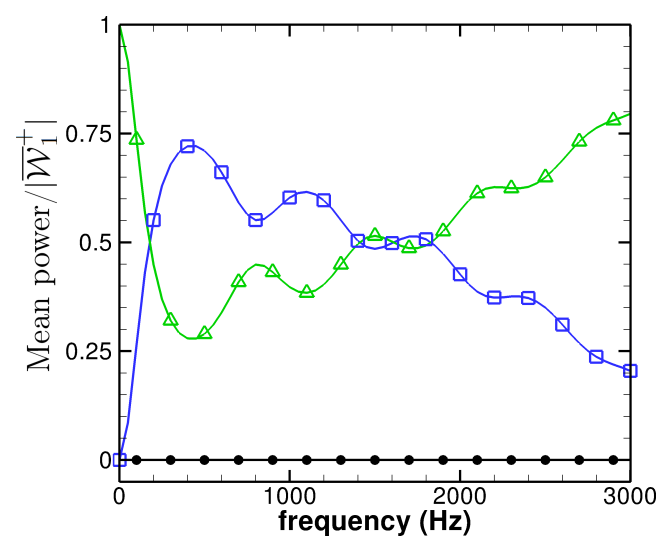

(a) upstream acoustic forcing $P_{1}^{+}$

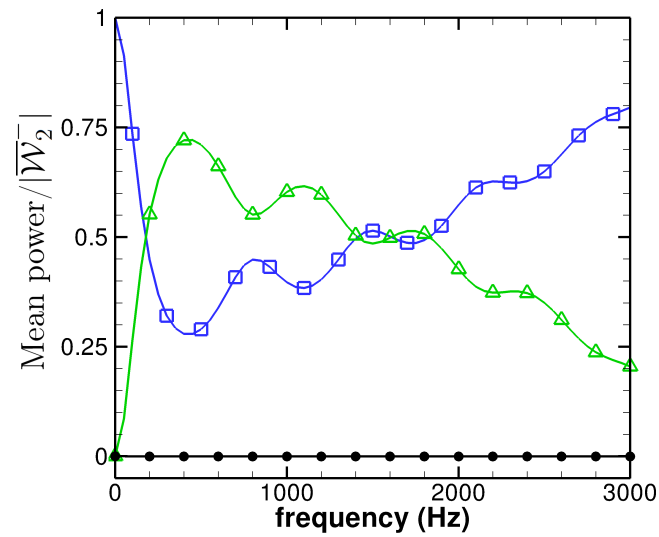

(b) downstream acoustic forcing $P_{2}^{-}$

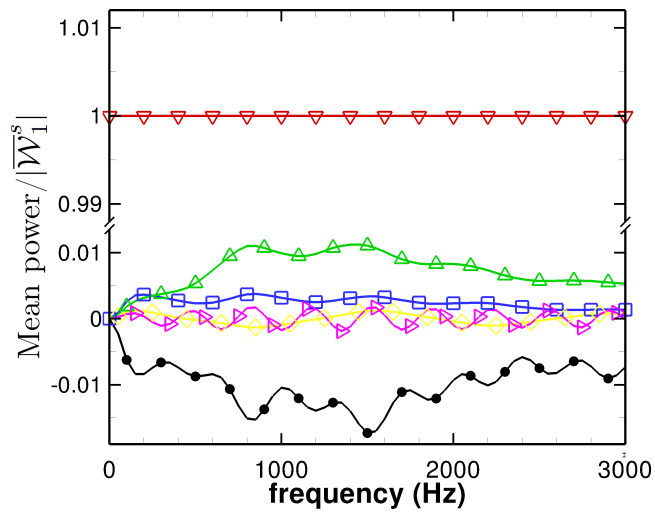

(c) entropy forcing $\sigma_{1}$

Fig. 9. Analytical evaluation of the contribution of the different terms to the energy balance for nozzle case T400_M07. $-\square \overline{\mathcal{W}}_{1}^{-} /\left|\overline{\mathcal{W}}_{f}\right|$, $\overline{\mathcal{W}}_{1}^{-, s} /\left|\overline{\mathcal{W}}_{f}\right|,-\triangle \overline{\mathcal{W}}_{2}^{+} /\left|\overline{\mathcal{W}}_{f}\right|,-\nabla \overline{\mathcal{W}}_{2}^{s} /\left|\overline{\mathcal{W}}_{f}\right|, \longrightarrow \overline{\mathcal{W}}_{2}^{+, s} /\left|\overline{\mathcal{W}}_{f}\right|,-\bullet \overline{\mathcal{D}} /\left|\overline{\mathcal{W}}_{f}\right|$

flux of the outgoing entropy wave is identical to the ingoing one, for all frequencies. This is obvious from Eq. (18) since, in the subsonic regime, upstream and downstream mean flows are identical and the entropy perturbation is simply convected by the mean flow without attenuation or amplification. Second, acoustic and interaction energy fluxes are much lower than entropy fluxes. Both fluxes $\overline{\mathcal{W}}_{1}^{-}$and $\overline{\mathcal{W}}_{2}^{+}$associated to the generated acoustic waves are positive, which is coherent with the associated energy leaving the nozzle. The downstream acoustic wave is more energetic than the upstream one, in agreement with the computed transfer functions of the nozzle (not reproduced here). To end, acoustic-entropy interaction terms $\overline{\mathcal{W}}_{1 / 2}^{ \pm, s}$ exhibit the lowest amplitudes and vary with frequency between positive and negative values, meaning that they either bring energy to or take energy from the nozzle flow, depending on the phase shift between the acoustic and entropy waves.

The contribution of the different waves is now reproduced in Fig. 10 for the choked case. For the upstream acoustic 


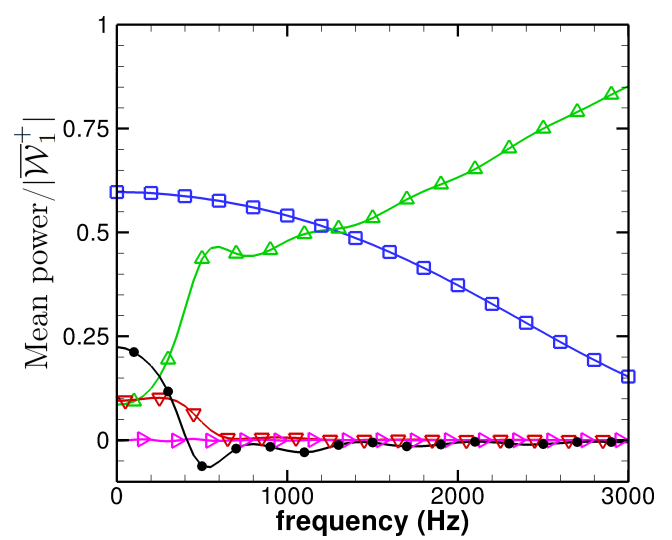

(a) upstream acoustic forcing $P_{1}^{+}$

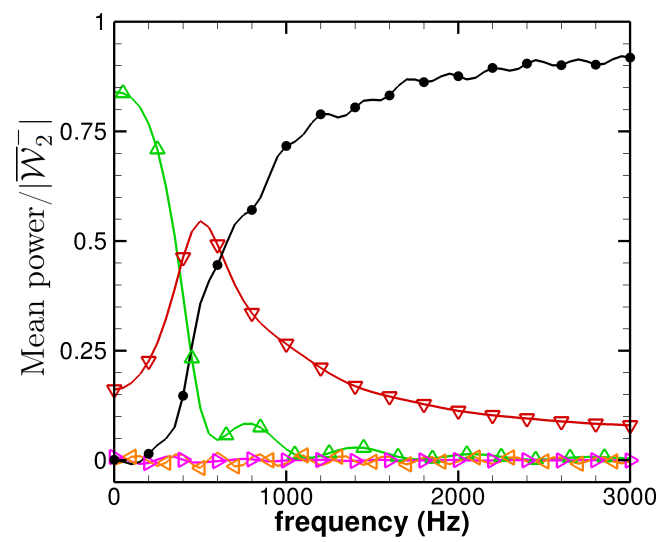

(b) downstream acoustic forcing $P_{2}^{-}$

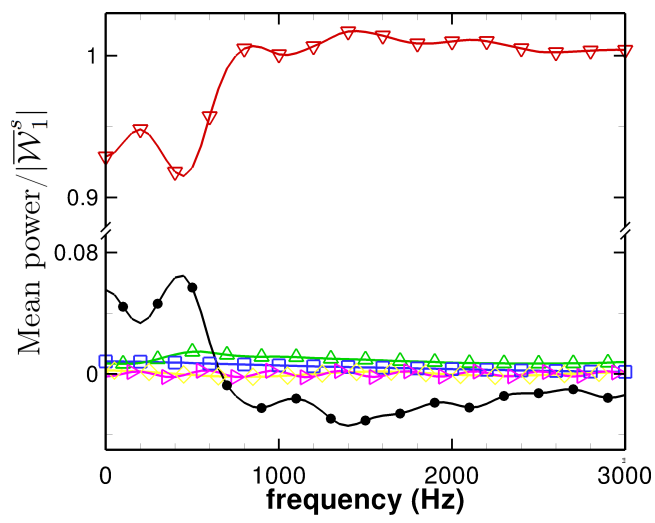

(c) entropy forcing $\sigma_{1}$

Fig. 10. Analytical evaluation of the contribution of the different terms to the energy balance for nozzle case T400_M10. $-\square \overline{\mathcal{W}}_{1}^{-} /\left|\overline{\mathcal{W}}_{f}\right|$, $\overline{\mathcal{W}}_{1}^{-, s} /\left|\overline{\mathcal{W}}_{f}\right|,-\triangle \overline{\mathcal{W}}_{2}^{+} /\left|\overline{\mathcal{W}}_{f}\right|,-\nabla \overline{\mathcal{W}}_{2}^{s} /\left|\overline{\mathcal{W}}_{f}\right|, \rightarrow \overline{\mathcal{W}}_{2}^{+, s} /\left|\overline{\mathcal{W}}_{f}\right|,-\triangleleft \overline{\mathcal{W}}_{2}^{-, s} /\left|\overline{\mathcal{W}}_{f}\right|,-\bullet \overline{\mathcal{D}} /\left|\overline{\mathcal{W}}_{f}\right|$.

forcing, Fig. 10 (a) indicates that at low frequencies the incoming energy is essentially reflected in the form of the retrograde acoustic wave. The dissipation is also quite important in the compact limit and the transmitted energy is distributed equally between the acoustic and the entropy wave. Fig. 10 (b) deals with the downstream acoustic forcing. At low frequencies, most of the acoustic energy is reflected and about $20 \%$ is converted into entropy fluctuation, with negligible dissipation. As the frequency increases, the reflected acoustic energy vanishes in agreement with the previous observations and the dissipation becomes predominant. An interesting feature appears near $500 \mathrm{~Hz}$, where dissipation and acoustic energy reflection are low and half the incoming acoustic energy is transferred to entropy fluctuations. Finally, the entropy-forced case is reproduced in Fig. 10 (c). The energy is essentially contained in the entropy fluctuations, which are slightly attenuated at low frequencies. As for the subsonic case, the energy of the acoustic fluctuations is low and is the most important for the downstream wave.

Huet 


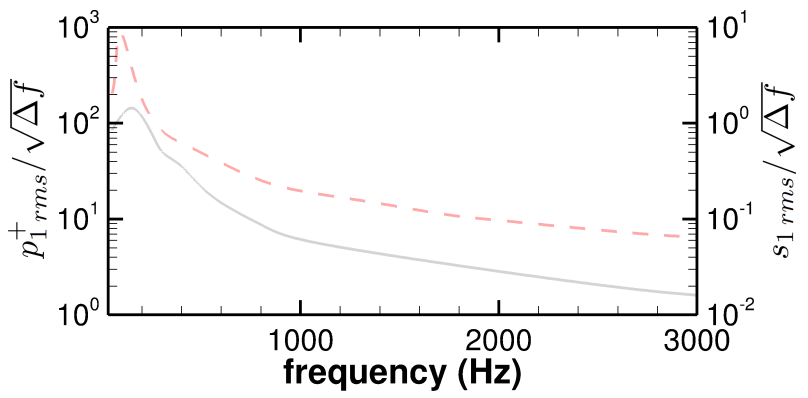

Fig. 11. Power spectral densities of the acoustic and entropy fluctuations generated by the flame inside the combustion chamber. $p_{1 r m s}^{+} / \sqrt{\Delta f},-s_{1 r m s} / \sqrt{\Delta f}$.

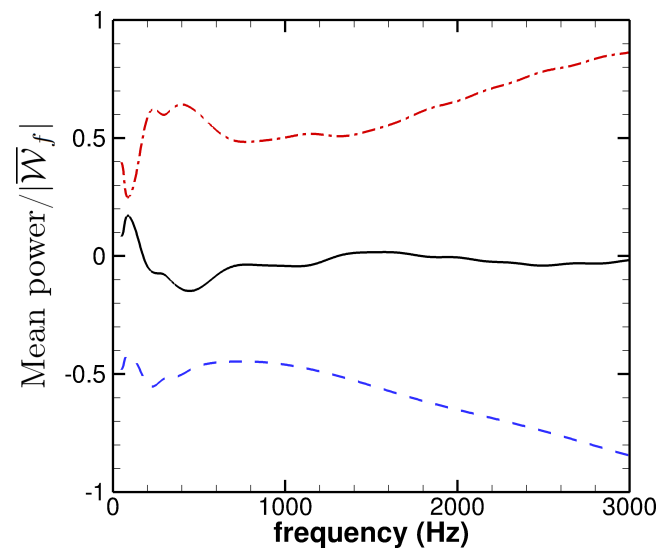

Fig. 12. Analytical mean disturbances energy budget for a real combustion noise source. $--\overline{\mathcal{W}}_{1} /\left|\overline{\mathcal{W}}_{f}\right|,-\cdot \overline{\mathcal{W}}_{2} /\left|\overline{\mathcal{W}}_{f}\right|,-\overline{\mathcal{D}}_{/} / \overline{\mathcal{W}}_{f} \mid$.

\section{Application to a real combustion noise source}

The last section of the paper illustrates the relevance of disturbances energy budgets for the investigation of a real combustion noise source. The model case considered is a combustor closed at its downstream extremity with the HAT nozzle at operating point T400_M10 of Tab. 1 . The power spectral densities of the acoustic $\left(p_{1 r m s}^{+}\right)$and entropy $\left(s_{1 \text { rms }}\right)$ forcings generated by the flame inside the combustor are reproduced in Fig. 11. They are idealized spectra taken from a reactive Large Eddy Simulation performed at ONERA [28] of the CESAM-HP test bench of EM2C laboratory, a pressurized combustor designed for lean premixed swirled combustion of gaseous propane $[38,39]$. In the absence of experimental and numerical data, acoustic forcing from the downstream end of the nozzle $\left(p_{2 r m s}^{-}\right)$is assumed to be nil. This is equivalent to consider the presence of an anechoic termination after the nozzle. This model case has no physical reality as the CESAM-HP bench is not closed in practice with the HAT nozzle and is operated at much higher temperatures, but its objective is to serve as an example of an actual application of the disturbances energy budgets to a practical case.

The mean disturbances energy budget of the model case is reproduced in Fig. 12, where the energy flux of the forcing contains contributions from acoustic and entropy fluctuations, $\overline{\mathcal{W}}_{f}=\overline{\mathcal{W}}_{1}^{+}+\overline{\mathcal{W}}_{1}^{s}+\overline{\mathcal{W}}_{1}^{+, s}$. In the compact limit, 50\% of the energy is reflected back to the combustion chamber, while $40 \%$ is transmitted and $10 \%$ is dissipated. As the frequency increases, most of the energy is transmitted through the nozzle and dissipation becomes negligible. To determine if the energy is of acoustic or entropic origin, that is to say if it is associated with noise or temperature fluctuations, one needs to evaluate the energy flux associated with each wave.

Figure 13 gives the detailed contribution of each wave to the energy budget. $96 \%$ of the forcing comes from the acoustic fluctuations and $4 \%$ from the entropy fluctuations, the interaction term $\overline{\mathcal{W}}_{1}^{+, s}$ being negligible. At low frequencies, half the incoming energy in reflected back to the combustion chamber in the form of a retrograde acoustic wave. An important fraction of the energy is also transmitted through the nozzle as an entropy fluctuation, particularly near $200 \mathrm{~Hz}$, while the flux associated with the downstream acoustic wave is limited to $20 \%$ of the incoming energy. As the frequency increases, the reflected part of the energy decreases and that associated with the downstream entropy fluctuation almost vanishes. Above $2000 \mathrm{~Hz}$, most of the energy is transmitted through the nozzle as a downstream acoustic wave. To end, the interaction terms are negligible for all frequencies. 


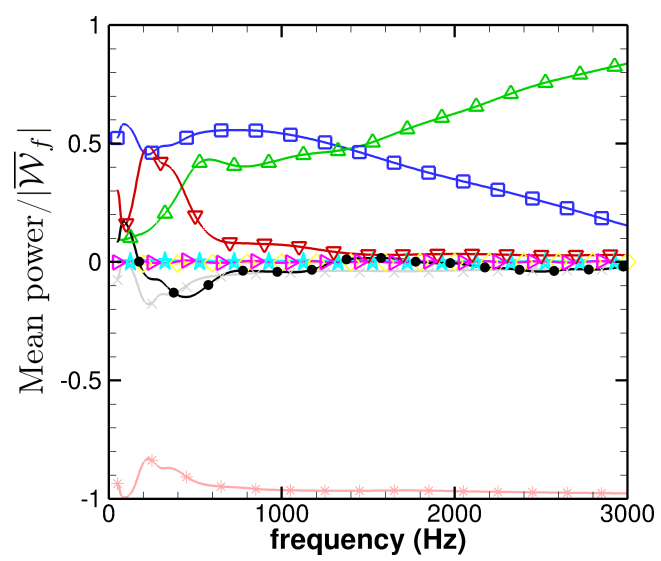

Fig. 13. Analytical evaluation of the contribution of the different terms to the energy balance for a real combustion noise source. $\overline{\mathcal{W}}_{1}^{+} /\left|\overline{\mathcal{W}}_{f}\right|,-\square \overline{\mathcal{W}}_{1}^{-} /\left|\overline{\mathcal{W}}_{f}\right|,-\times \overline{\mathcal{W}}_{1}^{s} /\left|\overline{\mathcal{W}}_{f}\right|,-\overline{\mathcal{W}_{1}} /\left|\overline{\mathcal{W}}_{f}\right|,-\overline{\mathcal{W}}_{1}^{-, s} /\left|\overline{\mathcal{W}}_{f}\right|,-\triangle \overline{\mathcal{W}}_{2}^{+} /\left|\overline{\mathcal{W}}_{f}\right|,-\nabla \overline{\mathcal{W}}_{2}^{s} /\left|\overline{\mathcal{W}}_{f}\right|,-\triangleright$ $\overline{\mathcal{W}}_{2}^{+, s} /\left|\overline{\mathcal{W}}_{f}\right|,-\bullet \overline{\mathcal{D}} /\left|\overline{\mathcal{W}}_{f}\right|$

\section{Conclusion}

The Hot Acoustic Test rig (HAT), a facility developed by DLR to investigate the noise generated by acoustic and entropy perturbations accelerated through a nozzle flow, is investigated analytically and numerically using quasi-1D approaches. Subsonic and choked regimes are considered. Low-order models are found to be very accurate and reproduce the experimental acoustic transmission and reflection coefficients with a very good accuracy.

To provide a better understanding on the transmission, reflection and dissipation of energy through the nozzle, energy budgets are investigated. The nonlinear disturbances energy initially proposed by Myers is rederived for quasi-1D flows and flux terms are linearised to separate the contributions of acoustic and entropy fluctuations. A limitation of this formulation, corresponding to a nonphysical negative dissipation -energy source-, is evidenced but is shown to have a negligible influence of the interpretation of the budgets. Dissipation is shown to remain low for all the considered cases, except for the choked nozzle with downstream acoustic forcing where a large part of the energy is dissipated by the shock at large frequencies, leading to a silent nozzle. Conversion of acoustic energy into entropy fluctuations also remains low for the choked configuration, with the exception of the low frequencies where the conversion may rise up to 50\%. Finally, the relevance of the disturbances energy budgets for the investigation of a real combustion noise source is illustrated for a model case combustor.

The reproduction of HAT experiment is a thorough validation of the low-order models of ONERA for the prediction of noise scattered through a nozzle. Disturbances energy moreover appears as a reliable tool for the investigation of energy scattering through such flows. When combined with a semi-analytical model it provides a fast estimation of the transmission, reflection and dissipation of perturbation energy as well as its conversion from acoustic to entropy or the inverse. Both the low-order models and the perturbations energy are useful tools for the investigation of indirect combustion noise. An extension to the turbine stage would be highly beneficial in order to deal with realistic aero-engines.

\section{Acknowledgements}

This work has been funded by ONERA. The Hot Acoustic Test rig (HAT) facility has been developed through the European Union Seventh Framework Programme (FP7/2007- 2013) within the framework of the RECORD project under grant agreement no. 312444. The author is very grateful to Dr. A. Giauque from École Centrale de Lyon for his help about disturbances energy and suggestions to improve the paper and to Drs. K. Knobloch and F. Bake from DLR for providing the experimental results.

\section{References}

[1] ACARE, 2010. Aeronautics and air transport: beyond vision 2020 (towards 2050).

[2] Dowling, A. P., and Mahmoudi, Y., 2015. "Combustion noise". Proceedings of the Combustion Institute, 35, pp. 65100.

[3] Candel, S., Durox, D., Ducruix, S., Birbaud, A.-L., Noiray, N., and Schuller, T., 2009. "Flame dynamics and combustion noise: progress and challenges". International Journal of Aeroacoustics, 8, pp. 1-56.

[4] Candel, S., 1972. "Analytical studies of some acoustic problems of jet engines". PhD thesis, California Institute of Technology. 
[5] Marble, F. E., 1973. "Acoustic disturbance from gas non-uniformities convecting through a nozzle". In Interagency Symposium on University Research in Transportation Noise, Stanford University, Stanford, CA, pp. 547-561.

[6] Morfey, C. L., 1973. "Amplification of aerodynamic noise by convected flow inhomogeneities". Journal of Sound and Vibration, 31, pp. 391-397.

[7] Marble, F. E., and Candel, S. M., 1977. "Acoustic disturbance from gas non-uniformities convected through a nozzle". Journal of Sound and Vibration, 55, pp. 225-243.

[8] Moase, W. H., Brear, M. J., and Manzie, C., 2007. "The forced response of choked nozzles and supersonic diffusers". Journal of Fluid Mechanics, 585, pp. 281-304.

[9] Giauque, A., Huet, M., and Clero, F., 2012. "Analytical analysis of indirect combustion noise in subcritical nozzles". $J$ Eng Gas Turb Powe, 134, p. 111202.

[10] Duran, I., and Moreau, S., 2013. "Solution of the quasi-one-dimensional linearized euler equations using flow invariants and the magnus expansion". Journal of Fluid Mechanics, 723, pp. 190-231.

[11] Huet, M., and Giauque, A., 2013. "A nonlinear model for indirect combustion noise through a compact nozzle". Journal of Fluid Mechanics, 733, pp. 268-301.

[12] Duran, I., and Morgans, A. S., 2015. "On the reflection and transmission of circumferential waves through nozzles". Journal of Fluid Mechanics, 773, pp. 137-153.

[13] Bake, F., Richter, C., Mühlbauer, B., Kings, N., Röhle, I., Thiele, F., and Noll, B., 2009. "The Entropy Wave Generator (EWG): A reference case on entropy noise". Journal of Sound and Vibration, 326, pp. 574-598.

[14] Kings, N., and Bake, F., 2010. "Indirect combustion noise: noise generation by accelerated vorticity in a nozzle flow". International Journal of Spray and Combustion Dynamics, 2, pp. 253-266.

[15] Mühlbauer, B., Noll, B., and Aigner, M., 2009. "Numerical investigation of the fundamental mechanism for entropy noise generation in aero-engines". Acta Acustica United With Acustica, 95, pp. 470-478.

[16] Leyko, M., Moreau, S., Nicoud, F., and Poinsot, T., 2011. "Numerical and analytical modelling of entropy noise in a supersonic nozzle with a shock". Journal of Sound and Vibration, 330, pp. 3944-3958.

[17] Lourier, J.-M., Huber, A., Noll, B., and Aigner, M., 2014. "Numerical analysis of indirect combustion noise generation within a subsonic nozzle". AIAA Journal, 52, pp. 2114-2126.

[18] Knobloch, K., Werner, T., and Bake, F., 2015. "Noise generation in hot nozzle flow". In Proceedings of ASME Turbo Expo 2015, Paper GT2015-43702.

[19] Knobloch, K., Werner, T., and Bake, F., 2015. "Entropy noise generation and reduction in a heated nozzle flow". In Proceedings of the 21st AIAA/CEAS Aeroacoustics Conference, Paper AIAA 2015-2818.

[20] Myers, M. K., 1991. "Transport of energy by disturbances in arbitrary steady flows". Journal of Fluid Mechanics, 226, pp. 383-400.

[21] Talei, M., Brear, M. J., Nicoud, F., Bodony, D. J., and Giauque, A., 2007. "Transport of disturbance energy in hot and cold turbulent jets". In Proceedings of the 13th AIAA/CEAS Aeroacoustics Conference, no. AIAA 2007-3633.

[22] Brear, M. J., Nicoud, F., Talei, M., Giauque, A., and Hawkes, E. R., 2012. "Disturbance energy transport and sound production in gaseous combustion". Journal of Fluid Mechanics, 707, pp. 53-73.

[23] Giauque, A., Poinsot, T., Brear, M., and Nicoud, F., 2006. "Budget of disturbance energy in gaseous reacting flows". In Proceedings of the Summer Program.

[24] Huet, M., 2016. "Nonlinear indirect combustion noise for compact supercritical nozzle flows". Journal of Sound and Vibration, 374, pp. 211-227.

[25] Giauque, A., 2007. "Fonctions de transfert de flamme et énergies de perturbation dans les écoulements réactifs". $\mathrm{PhD}$ thesis, Institut National Polytechnique de Toulouse, France.

[26] Knobloch, K., Neuhaus, L., Bake, F., and Paolo Gaetani, G. P., 2016. "Experimental assessment of noise generation and transmission in a high-pressure transonic turbine stage". In Proceedings of ASME Turbo Expo 2016, Paper GT201657209.

[27] Knobloch, K., Holewa, A., Guérin, S., Mahmoudi, Y., Hynes, T., and Bake, F., 2016. "Noise transmission characteristics of a high pressure turbine stage". In Proceedings of the 22nd AIAA/CEAS Aeroacoustics Conference, Paper AIAA 2016-3001.

[28] Huet, M., Vuillot, F., Bertier, N., Mazur, M., Kings, N., Tao, W., Scouflaire, P., Richecoeur, F., Ducruix, S., Lapeyre, C., and Poinsot, T., 2016. "Recent improvements in combustion noise investigation: from the combustion chamber to nozzle flow". Aerospace Lab, 11.

[29] Bogey, C., and Bailly, C., 2004. "A family of low dispersive and low dissipative explicit schemes for flow and noise computations". Journal of Computational Physics, 194, pp. 194-214.

[30] Bogey, C., de Cacqueray, N., and Bailly, C., 2009. "A shock-capturing methodology based on adaptative spatial filtering for high-order non-linear computations". Journal of Computational Physics, 228, pp. 1447-1465.

[31] Poinsot, T. J., and Lele, S. K., 1992. "Boundary conditions for direct simulations of compressible viscous flows". Journal of Computational Physics, 101, pp. 104-129.

[32] Huet, M., 2015. "One-dimensional characteristic boundary conditions using nonlinear invariants". Journal of Compu- 
tational Physics, 283, pp. 312-328.

[33] Giauque, A., Huet, M., Clero, F., Ducruix, S., and Richecoeur, F., 2013. "Thermoacoustic shape optimization of a subsonic nozzle". J Eng Gas Turb Powe, 135, p. 102601.

[34] Huet, M., 2017. "Influence of calorically perfect gas assumption and thermal diffusion on indirect noise generation". In Proceedings of the 24th International Congress on Sound and Vibration.

[35] Zheng, J., Huet, M., Cléro, F., Giauque, A., and Ducruix, S., 2015. "A 2D-axisymmetric analytical model for the estimation of indirect combustion noise in nozzle flows". In Proceedings of the 21st AIAA/CEAS Aeroacoustics Conference, no. AIAA 2015-2974.

[36] Zheng, J., 2016. "Analytical and numerical study of the indirect combustion noise generated by entropy disturbances in nozzle flows". PhD thesis, Université Paris-Saclay.

[37] Emmanuelli, A., Huet, M., Le Garrec, T., and Ducruix, S., 2017. "CAA study of entropy noise in nozzle flow for the validation of a 2D semi-analytical model". In Proceedings of the ASME Turbo Expo 2017, Paper GT2017-63640.

[38] Mazur, M., Tao, W., Scouflaire, P., Richecoeur, F., and Ducruix, S., 2015. "Experimental and analytical study of the acoustic properties of a gas turbine model combustor with a choked nozzle". In Proceedings of ASME Turbo Expo, Paper GT2015-43013.

[39] Lapeyre, C. J., Mazur, M., Scouflaire, P., Richecoeur, F., Ducruix, S., and Poinsot, T., 2016. "Acoustically induced flashback in a staged swirl-stabilized combustor". Flow, Turbulence and Combustion. 